\title{
Autonomic Protocol and Architecture for Devices in Internet of Things
}

\author{
Qazi Mamoon Ashraf ${ }^{1}$, Mohamed Hadi Habaebi ${ }^{2}$, Gopinath Rao Sinniah ${ }^{3}$, Musse Mohamud \\ Ahmed $^{4}$, Sheroz Khan ${ }^{5}$, Shihab Hameed ${ }^{6}$ \\ 1,2,4,5,6 Department of Electrical and Computer Engineering, International Islamic University Malaysia \\ ${ }^{3}$ Internet of Things, Wireless Communication, MIMOS BHD
}

Email: ${ }^{1}$ mamoonq@gmail.com, ${ }^{2}$ habaebi@iium.edu.my, ${ }^{3}$ gopinath.rao@mimos.my, ${ }^{4}$ musse@iium.edu.my,
sheroz@iium.edu.my, ${ }^{6}$ shihab@iium.edu.my

\begin{abstract}
With the advancement taking place in communication technologies, Internet of Things (IoT) is expected to play a major role in realizing smartness and functionality needed for applications such as Smart Homes and Electrical Smart Grids. However, in order to achieve that far reaching goal, select concepts of computing autonomy have to be suitably ported to IoT architecture. Recently reported works describe various frameworks, models and protocols for computing autonomy. Some others focus more on the practical implementation of autonomy using self-star (self-*) behavior in end nodes. This work proposes an autonomic architecture for IoT based on IBM's autonomic framework. An accompanying protocol for service communication is also presented. Preliminary tests prove its suitability in an IoT environment from the perspective of energy conservation for the network.
\end{abstract}

Index Terms-Internet of Things, Scalability, Wireless Sensor Networks, Contiki, Single-Hop, Autonomy, Smart Grids

\section{INTRODUCTION}

During the last three decades, tremendous research on communication for computers has led to the growth of Internet of Things (IoT) where traditional Internet is being extended to include diverse objects [1] [2]. The main vision behind IoT is that electronic devices, ranging from smart phones to embedded devices, are becoming IP enabled. This enables devices to compute, organize and then communicate with other communicating stations or similar devices via the Internet. The IoT is setup and maintained economically and energy-efficiently through sensors attached to these objects. IoT is thus a collection of simple devices, efficient protocols, and resourceful web services [3].

An important requirement of IoT is that its constituent components should achieve organizational structure without human intervention. Organizational structure would refer to the automatic setup of network topology and dynamic nature of the functionality. Computing world translates such a concept to autonomy. Autonomy in IoT thus refers to selfconfiguration where the network must be able to adapt dynamically and adjust the offered services. This could either refer to initial registration, setup and handshake of the nodes, or it could refer to changes in routes for multihop setups. Thus, autonomy warrants for dynamic adjustments to be done automatically to component configuration for best handling changing situations. Self-configuration aims to achieve organization of these IoT smart objects by autonomic and self-star (self-*) principles. Here self-star refers to the set of selfadapting, self-configuring, self-healing and selforganizing set of requirements. This paradigm translates directly to the fulfilment of autonomy. Thus, the aim of self-* principles is to reduce the need for manual intervention and management at every level as much as would be possible. A self-configuring system must configure and reconfigure itself under varying and unpredictable conditions.

This paper is organized as follows. Section II presents the related work and the motivation behind this research. Section III presents the design of the architecture and the associated communication protocol. Section IV presents preliminary results followed by a short discussion in Section V.

\section{RELATED WORK}

IBM [4] has introduced a framework of autonomic computing which is aimed to make the management of systems easier. The framework consists of two functionally important entities, namely, (1) Managed Resource and (2) Autonomic Manager. The managed resource is the less complex entity. In an IoT setup, the

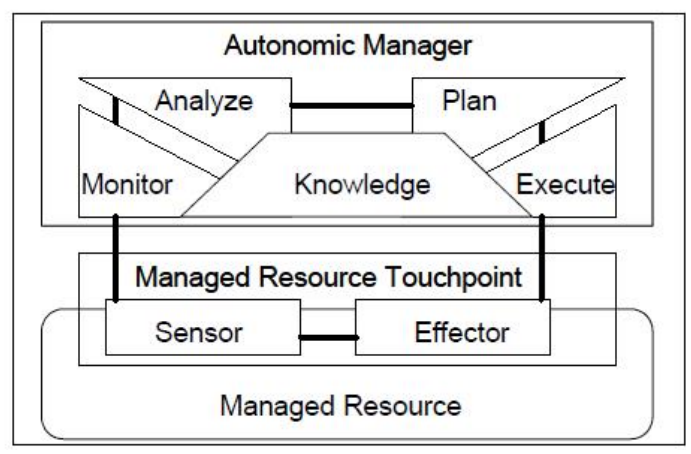

Figure 1: IBM Autonomic Control Loop [4] 